Journal of mathematics and computer Science 15 (2015) 159 - 166

Contents list available at JMCS
Journal Homepage: www.tjmcs.com

\title{
Development of Organizational Strategies by Fuzzy Logic and MCDM (FMCDM) \\ Case Study: Distribution of Book of Marv Eghlim Roshan Company
}

\author{
M. Nasrabadi ${ }^{1}$, M. A. Sobhanallahi \\ ${ }^{1}$ Department of Industrial Engineering, Kharazmi University, Tehran, Iran \\ nasrabadi.mehri1392@gmail.com
}

Article history:

Received November 2014

Accepted February 2015

Available online February 2015

\begin{abstract}
One of the important factors for success an organization is Utilization and adoption appropriate strategies with regard to internal and external conditions of organization. In this paper is used the SWOT matrix for evaluation of effective internal and external factors on performance of organization. In addition to fuzzy techniques and Linguistic variables is used for the weighting of the criteria, Since the criteria (internal and external factors) are associated with some ambiguities and uncertainties. Finally, we used OWA method (ordered weighted average) for ranking of strategies.
\end{abstract}

Keywords: SWOT matrix، FMCDM، Group - Fuzzy MADM

\section{Introduction}

Nowadays, Have a competitive advantage is considered as an effective factor in advancement an organization. Organizations have to use varied methods to gain competitive advantage, that correct selection of strategies is one of them. Strategies are comprehensive programs that included activities and prepare resources to achieve to goals. Resources of organization is limited, so organization manager have to use effective methods for selecting the best strategy.There are afew paper for development of strategies, For example, Kurttila [2000] proposed a hybrid method for limits of measurement and evaluation of swot matrix. Swanson [2001] reviewed the relationship between maintenance strategies and performance. Mechefske \& wang [2003] used linguistic variables for selecting control strategies. Dyson [2004] used SWOT method. Yuksel \& Dagdeviren [2007] used ANP method for designing and development of SWOT strategies. Sevkli [2012] proposed a fuzzy ANP model, according to SWOT analysis. In this paper is used SWOT matrix for evaluation of internal and external factors affecting on performance of organization, and FMCDM for rattting strategies. Most existing models are used ANP method and AHP method for ratting of strategies. In this paper, we use OWA method for ratting of strategies. OWA method determines ratting with expressing mental characteristics of decision makers. 


\section{SWOT matrix}

\begin{tabular}{|c|c|c|}
\hline Strengths & Opportunities & Threats \\
\hline Weaknesses & $\begin{array}{c}\text { It's the best time for } \\
\text { having an operation } \\
\text { Conditions and Overcame } \\
\text { the Weaknesses of the } \\
\text { organization }\end{array}$ & $\begin{array}{c}\text { We need to turn threats } \\
\text { into opportunities }\end{array}$ \\
\hline \multicolumn{2}{|c|}{ We should use defensive } \\
strategies
\end{tabular}

\section{Multi - criteria decision - making (MCDM)}

Multiple- criteria decision- making is a sub-discipline of operations research that explicitly considers multiple criteria in decision-making environments. Whether in our daily lives or in professional settings, there are typically multiple conflicting criteria that need to be evaluated in making decisions.Some MCDM methods are TOPSIS, GP, AHP, ANP, SAW, OWA.

\subsection{The ordered weighted average method}

This method determines risk taker and risk averse decision makers.

OWA operator of dimension $\mathrm{n}$ is a mapping $F: R_{\mathrm{n}}: \rightarrow R$ that has an associated collection of weights.

$$
W=\left[w_{1}, \ldots, w_{\mathrm{n}}\right]
$$

$\mathrm{W}$ vector lying in the unit interval and summing to one and with

$F\left(a_{1}, \ldots, a_{\mathrm{n}}\right)=\sum w_{\mathrm{j}} b_{\mathrm{j}}$, where $b_{\mathrm{j}}$ is the $j^{\text {th }}$ largest of the $\mathrm{a}_{\mathrm{i}}$.

By choosing different $W$ one can implement different aggregation operators. The OWA operator is a non-linear operator as a result of the process of determining the $b_{\mathrm{j}}$.

Two features have been used to characterize the OWA operators. The first is the attitudinal character (orness).

This is defined as:

$$
\begin{gathered}
A-C(W)=\frac{1}{n-1} \sum_{j=1}^{n}(n-j) w_{j} . \\
A-C(W) \in[0,1] .
\end{gathered}
$$

In addition $\mathrm{A}-\mathrm{C}(\max )=1, \mathrm{~A}-\mathrm{C}($ ave $)=\mathrm{A}-\mathrm{C}($ med $)=0.5$ and $\mathrm{A}-\mathrm{C}(\min )=0$. Thus the $\mathrm{A}-\mathrm{C}$ goes from 1 to 0 as we go from Max to Min aggregation. The attitudinal character characterizes the similarity of aggregation to OR operation (OR is defined as the Max).

The weight is calculated through of the following method. 


$$
W i=Q(i / n)-Q(i-1 / n) \quad, i=1, \ldots, n
$$

$\mathrm{n}$ is the number of the indexes and $\mathrm{i}$ is the count of the indexes.

$$
\begin{gathered}
Q(r)=\left\{\begin{array}{cc}
0 & \text { if } r<a \\
\frac{r-a}{b-a} & \text { if } a \leq r \leq b \\
1 & \text { if } r>b
\end{array}\right. \\
a \geq 0, \quad Q(r)=r \alpha
\end{gathered}
$$

The function $f$ is related to the degree of optimism.

$$
\begin{gathered}
\int_{0}^{1} Q(r) d r=\int_{0}^{1} r^{\alpha} d r=\frac{1}{\alpha+1} \\
A-C(W)<0.5 \rightarrow 1>\alpha
\end{gathered}
$$

DM is risk averse.

$$
\alpha=1 \rightarrow A-C(W)=0.5
$$

DM is neutral

$\mathrm{DM}$ is risk taking

$$
\alpha<1 \quad \rightarrow \quad A-C(W)>0.5
$$

\section{The construction of fuzzy decision matrix}

$$
\begin{gathered}
\mathrm{D}=\left[\begin{array}{ccc}
X_{11} & \cdots & X_{1 n} \\
\vdots & \ddots & \vdots \\
X_{m 1} & \cdots & X_{m n}
\end{array}\right] \\
W=\left[w_{1}, w_{2}, \ldots w_{\mathrm{n}}\right] \\
X_{-}(i j=1 / K)[X i j 1(+) X i j 2(+) \ldots(+) X i j k]
\end{gathered}
$$




\subsection{Scale-up the fuzzy decision matrix with linear method}

$\mathrm{B}$ is the Set of the positive measures and $\mathrm{C}$ is the Set of the negative measures.

$$
\begin{gathered}
\boldsymbol{R}=\left[r_{i j}\right]_{m \times n} \\
r_{i j}=\left(\frac{a_{i j}}{c_{j}^{*}}, \frac{b_{i j}}{c_{j}^{*}}, \frac{c_{i j}}{c_{j}^{*}}\right), \quad j \in B \\
C j *=\max c i j \quad \text { if } \quad j \in B \\
r i j=\left(\frac{a_{j}}{c_{i j}}, \frac{a_{j}}{b_{i j}}, \frac{a_{j}}{a_{i j}}\right), \quad j \in C \\
a j=\min a i j \quad \text { if } \quad j \in C
\end{gathered}
$$

4.2. Construction of Scale up weighted matrix

$$
\begin{aligned}
& {\left[v_{-} i j\right]_{-}(m \times n) \quad i=1,2, \ldots, m \quad j=1,2, \ldots, n} \\
& v_{-} i j=r_{-}\left(i j(.) w_{-} j\right)
\end{aligned}
$$

\subsection{Linguistic variables for weighting}

\begin{tabular}{|c|c|}
\hline Weight & Fuzzy Set \\
\hline Very Low(VL) & $(\mathbf{0 , 0 , 0 . 1 )}$ \\
\hline Low(L) & $\mathbf{( 0 , 0 . 1 , 0 . 3 )}$ \\
\hline Medium Low(ML) & $(\mathbf{0 . 1 , 0 . 3 , 0 . 5 )}$ \\
\hline Medium(M) & $\mathbf{( 0 . 3 , 0 . 5 , 0 . 7 )}$ \\
\hline Medium High(MH) & $\mathbf{( 0 . 5 , 0 . 7 , 0 . 9 )}$ \\
\hline High(H) & $(\mathbf{0 . 7 , 0 . 9 , 1 )}$ \\
\hline Very High(VH) & $(\mathbf{0 . 9}, 1,1)$ \\
\hline
\end{tabular}




\subsection{Linguistic variables for ranking}

\begin{tabular}{|c|c|}
\hline Ranking & Fuzzy sets \\
\hline Very Poor (VP) & $(\mathbf{0 , 0 , 1 )}$ \\
\hline poor(P) & $(\mathbf{0 , 1 , 3 )}$ \\
\hline Medium Poor (MP) & $(\mathbf{1 , 3 , 5})$ \\
\hline Fair (F) & $(\mathbf{3 , 5 , 7 )}$ \\
\hline Medium good(MG) & $\mathbf{( 5 , 7 , 9 )}$ \\
\hline Good (G) & $\mathbf{( 7 , 9 , 1 0 )}$ \\
\hline Very good (VG) & $\mathbf{( 9 , 1 0 , 1 0 )}$ \\
\hline
\end{tabular}

\section{Case study}

We use OWA method for development of strategies in distribution of book Company.

We considers two $D M s, k=2$.

Strengths are $s_{1}, s_{2}, s_{3}$.

Weaknesses are $w_{1}, w_{2}, w_{3}$.

Opportunity is $O_{1}$.

Threats are $t_{1}, t_{2}$.

The Suggested strategies are $s o_{1}, w o_{1}, s t_{1}, s t_{2}, w t_{1}$.

\subsection{The internal and external factors affecting on the performance of the company}

\begin{tabular}{|c|c|c|}
\hline $\begin{array}{c}\text { Weakness } \\
\text { 1- poor marketing and } \\
\text { don't use of Technology. } \\
\text { 2-Inappropriate Location } \\
\begin{array}{c}\text { of Company. } \\
\text { 3-Lack of expert personnel } \\
\text { and interested in working. }\end{array}\end{array}$ & $\begin{array}{c}\text { Strenght } \\
\text { 1-distribution of book to } \\
\text { Most cities and adjacent } \\
\text { provinces } \\
\text { 2-diversity of Book } \\
\text { 3- reputation of Company }\end{array}$ & \\
\hline $\begin{array}{c}\text { WO } \\
\text { 1-employmenting some of } \\
\text { skilled marketer }\end{array}$ & $\begin{array}{c}\text { SO } \\
\text { 1- Establishment of } \\
\text { Various exhibitions in } \\
\text { different areas of the city }\end{array}$ & Opportunity \\
\hline $\begin{array}{c}\text { ST } \\
\text { WT }\end{array}$ & $\begin{array}{c}\text { 2-If you buy some } \\
\text { books,you will receive } \\
\text { amount of discounts }\end{array}$ & Threat \\
\hline 1-lending of book & \multicolumn{2}{|c|}{} \\
\hline
\end{tabular}




\subsection{Comments of DMs about the impact of the strategies on the internal and external factors}

\begin{tabular}{|c|c|c|c|c|c|c|c|c|c|c|}
\hline & \multicolumn{2}{|c|}{$\mathbf{S O}_{1}$} & \multicolumn{2}{|c|}{$\mathrm{SO}_{2}$} & \multicolumn{2}{|c|}{$\mathrm{WO}_{1}$} & \multirow{2}{*}{$\frac{\mathbf{S T}_{\mathbf{1}}}{\mathrm{D} 1}$} & \multirow{2}{*}{$\frac{\mathbf{S T}_{\mathbf{2}}}{\mathrm{D} 2}$} & \multicolumn{2}{|c|}{$\mathbf{W T}_{1}$} \\
\hline & D1 & D2 & D1 & D2 & D1 & D2 & & & D1 & $\mathrm{D} 2$ \\
\hline $\mathbf{O}_{1}$ & $\mathrm{VH}$ & $\mathrm{H}$ & $\mathrm{H}$ & $\mathrm{H}$ & $\mathrm{H}$ & $\mathrm{VH}$ & $\mathrm{H}$ & $\mathrm{H}$ & $\mathrm{H}$ & $\mathrm{VH}$ \\
\hline $\mathbf{T}_{1}$ & $\mathrm{H}$ & $\mathrm{H}$ & $\mathrm{H}$ & MH & $\mathrm{H}$ & $\mathrm{H}$ & $\mathrm{H}$ & $\mathrm{VH}$ & $\mathrm{H}$ & $\mathrm{H}$ \\
\hline $\mathbf{T}_{1}$ & VL & $\mathrm{L}$ & $\mathrm{L}$ & VL & M & MH & $\mathrm{H}$ & $\mathrm{H}$ & VH & VH \\
\hline $\mathbf{S}_{1}$ & M & ML & $\mathrm{H}$ & $\mathrm{VH}$ & MH & M & MH & MH & $\mathrm{L}$ & VL \\
\hline $\mathbf{S}_{2}$ & $\mathrm{VH}$ & $\mathrm{H}$ & $\mathrm{H}$ & $\mathrm{H}$ & MH & M & $\mathrm{H}$ & $\mathrm{MH}$ & $\mathrm{MH}$ & $\mathrm{H}$ \\
\hline $\mathbf{S}_{\mathbf{3}}$ & MH & M & $\mathrm{M}$ & MH & M & $\mathrm{M}$ & ML & ML & $\mathrm{M}$ & ML \\
\hline $\mathbf{W}_{1}$ & $\mathrm{H}$ & $\mathrm{H}$ & $\mathrm{H}$ & $\mathrm{H}$ & $\mathrm{M}$ & ML & $\mathrm{H}$ & $\mathrm{MH}$ & $\mathrm{MH}$ & $\mathrm{MH}$ \\
\hline $\mathbf{W}_{2}$ & MH & $\mathrm{H}$ & MH & $\mathrm{MH}$ & ML & M & ML & M & M & MH \\
\hline $\mathbf{W}_{3}$ & ML & ML & $\mathrm{H}$ & $\mathrm{MH}$ & ML & ML & $\mathrm{L}$ & $\mathrm{L}$ & $\mathrm{L}$ & ML \\
\hline
\end{tabular}

\section{3. construction of Fuzzy decision matrix}

\begin{tabular}{|c|c|c|c|c|c|c|c|c|c|}
\hline $\begin{array}{l}\begin{array}{l}\text { Strate } \\
\text { gies }\end{array} \\
\text { Criteki } \\
\text { a }\end{array}$ & $\mathrm{O}_{1}$ & $\mathrm{~T}_{1}$ & $\mathrm{~T}_{2}$ & $\mathrm{~S}_{1}$ & $\mathrm{~S}_{2}$ & $\mathrm{~S}_{3}$ & $\mathrm{~W}_{1}$ & $\mathrm{~W}_{2}$ & $\mathrm{~W}_{3}$ \\
\hline Index & + & - & - & + & + & + & - & - & - \\
\hline $\mathrm{SO}_{1}$ & $\begin{array}{l}(0.8 .0 .95 . \\
1)\end{array}$ & $(0.7,0.9,1)$ & $\begin{array}{l}(0.15,0.3,0 . \\
5)\end{array}$ & )$^{(0.2,0.4,0.6}$ & $\begin{array}{l}(0.8 .0 .95 . \\
1)\end{array}$ & $\begin{array}{l}(0.4 \cdot 0.6,0.8 \\
)^{2}\end{array}$ & $(0.7,0.9,1)$ & $(0.1,0.3,0.5)$ & $\begin{array}{l}(0.6,0.8,0.9 \\
5)\end{array}$ \\
\hline $\mathrm{WO}_{1}$ & $\begin{array}{l}(0.7,0.9,1 \\
)^{2}\end{array}$ & $\begin{array}{l}(0.6,0.8,0.9 \\
5)\end{array}$ & $\begin{array}{l}0.05,0.2,0.4 \\
)(\end{array}$ & $(0.8 .0 .95 .1)$ & $0.7,0.9,1)$ & $\begin{array}{l}(0.4 \cdot 0.6,0.8 \\
)\end{array}$ & $(0.7,0.9,1)$ & $(0.6,0.8,0.95)$ & $\begin{array}{l}(0.5,0.7,0.9 \\
)\end{array}$ \\
\hline $\mathrm{ST}_{1}$ & $\begin{array}{l}(0.8 .0 .95 \\
1)\end{array}$ & $(0.7,0.9,1)$ & $\begin{array}{l}(0.6,0.8,0.9 \\
5)\end{array}$ & $\begin{array}{l}(0.4 \cdot 0.6,0.8 \\
)^{2}\end{array}$ & $\begin{array}{l}(0.4 .0 .6,0 . \\
8)\end{array}$ & $\begin{array}{l}(0.3,0.5,0.7 \\
)^{2}\end{array}$ & $(0.2,0.4,0.6)$ & $(0.1,0.3,0.5)$ & )$^{(0.2,0.4,0.6}$ \\
\hline $\mathrm{ST}_{2}$ & $\begin{array}{l}(0.7,0.9,1 \\
)^{\prime}\end{array}$ & $\begin{array}{l}(0.6,0.63,0 . \\
75)\end{array}$ & $\begin{array}{l}(0.05 .0 .05,0 \\
.07)\end{array}$ & $\begin{array}{l}(0.5,0.7,0.9 \\
)^{2}\end{array}$ & $\begin{array}{l}(0.6,0.8,0 . \\
95)\end{array}$ & $\begin{array}{l}(0.13,0.38,0 \\
.63)\end{array}$ & $\begin{array}{l}(0.21,0.25,0 \\
.33)\end{array}$ & $(0.13,0.26,1)$ & $(0.33,0.5,1)$ \\
\hline $\mathrm{WT}_{1}$ & $\begin{array}{l}(0.8 .0 .95 . \\
1)\end{array}$ & $\begin{array}{l}(0.6,0.6,0.6 \\
7)\end{array}$ & $\begin{array}{l}(0.05,0.05,0 \\
.05)\end{array}$ & $(0,0.05,0.2)$ & $\begin{array}{l}(0.6,0.8,0 . \\
95)\end{array}$ & $\begin{array}{l}(0.25,0.5,0 . \\
75)\end{array}$ & $\begin{array}{l}(0.22,0.25,0 \\
.33)\end{array}$ & $(0.13,0.26,1)$ & $\begin{array}{l}(0.25,0.33,0 \\
.5)\end{array}$ \\
\hline
\end{tabular}




\subsection{Scale-up Fuzzy decision matrix with linear method}

\begin{tabular}{|c|c|c|c|c|c|c|c|c|c|}
\hline Criteria & $\mathrm{O}_{1}$ & $\mathrm{~T}_{1}$ & $\mathrm{~T}_{2}$ & $\mathrm{~S}_{1}$ & $\mathrm{~S}_{2}$ & $\mathrm{~S}_{3}$ & $\mathrm{~W}_{1}$ & $\mathrm{~W}_{2}$ & $\mathrm{~W}_{3}$ \\
\hline Index & + & - & - & + & + & + & - & - & - \\
\hline $\mathrm{SO}_{1}$ & $(0.8 .0 .95 .1)$ & $(0.6,0.67 .086$ & $(0.1,0.17,0.33)$ & $(0.2,0.4,0.6)$ & $(0.8 .0 .95 .1)$ & $(0.5,0.75,1)$ & $\begin{array}{l}(0.2,0.22,0 \\
29)\end{array}$ & $\begin{array}{l}(0.11,0.17,0 . \\
5)\end{array}$ & $(0.21,0.25,0.33)$ \\
\hline $\mathrm{WO}_{1}$ & $(0.7,0.9,1)$ & $(0.63,075,1)$ & $(0.13,0.25,1)$ & $(0.8 .0 .95 .1)$ & $0.7,0.9,1)($ & $(0.5,0.75,1)$ & $\begin{array}{l}(0.2,0.22,0 . \\
29)\end{array}$ & $\begin{array}{l}(0.05,0.06,0 . \\
08)\end{array}$ & $(0.22,0.29,0.4)$ \\
\hline $\mathrm{ST}_{1}$ & $(0.8 .0 .95 .1)$ & $\begin{array}{l}(0.6,0.67, .08 \\
6)\end{array}$ & $\begin{array}{l}(0.05,0.06,0.0 \\
8)\end{array}$ & $(0.4 .0 .6,0.8)$ & $(0.4 .0 .6,0.8)$ & $\begin{array}{l}(0.38,0.63,088 \\
)\end{array}$ & $(0.33,0.5,1)$ & $\begin{array}{l}(0.11,0.17,0 . \\
5)\end{array}$ & $(0.33,0.5,1)$ \\
\hline $\mathrm{ST}_{2}$ & $(0.7,0.9,1)$ & $\begin{array}{l}(0.6,0.63,0.7 \\
5)\end{array}$ & $\begin{array}{l}(0.05 .0 .05,0.0 \\
7)\end{array}$ & $(0.5,0.7,0.9)$ & $\begin{array}{l}(0.6,0.8,0.95 \\
)\end{array}$ & $\begin{array}{l}(0.13,0.38,0.6 \\
3)\end{array}$ & $\begin{array}{l}(0.21,0.25,0 \\
.33)\end{array}$ & $(0.13,0.26,1)$ & $(0.33,0.5,1)$ \\
\hline $\mathrm{WT}_{1}$ & $(0.8 .0 .95 .1)$ & $\begin{array}{l}(0.6,0.6,0.67 \\
)\end{array}$ & $\begin{array}{l}(0.05,0.05,0.0 \\
5)\end{array}$ & $(0,0.05,0.2)$ & $\begin{array}{l}(0.6,0.8,0.95 \\
)\end{array}$ & $(0.25,0.5,0.75)$ & $\begin{array}{l}(0.22,0.25,0 \\
.33)\end{array}$ & $(0.13,0.26,1)$ & $(0.25,0.33,0.5)$ \\
\hline
\end{tabular}

\subsection{Calculation of the Weights of the criteria}

We calculated the weights of the criteria with relations of 1 and 6 . Then we calculated value of each option with OWA operator and relation1.

\subsection{Score to options}

\begin{tabular}{|c|c|c|c|c|c|c|}
\hline & $\alpha$ & $\mathrm{SO}_{1}$ & $\mathrm{WO}_{1}$ & $\mathrm{ST}_{1}$ & $\mathrm{ST}_{2}$ & $\mathrm{WT}_{1}$ \\
\hline risk taking & 0.1 & $(0.73,0.89,0.94)$ & $(0.74,0.88,0.97)$ & $(0.73,0.87,0.97)$ & $(0.65,0.83,0.96)$ & $(0.72,0.86,0.94)$ \\
& 0.6 & $(0.55,0.63,0.66)$ & $(0.54,0.7,0.57)$ & $(0.48,0.64,0.85)$ & $(0.54,0.69,0.81$ & $(0.46,0.56,0.7)$ \\
\hline Neutral & 1 & $(0.73,0.89,0.94)$ & $(0.74,0.88,0.97)$ & $(0.32,0.47,0.7)$ & $(0.37,0.5,0.74)$ & $(0.33,0.44,0.6)$ \\
& & $(0.55,0.63,0.66)$ & $(0.54,0.7,0.57)$ & & & \\
\hline risk averse & 1.5 & $(0.31,0.42,0.58)$ & $(0.33,0.47,0.68)$ & $(0.32,0.46,0.73)$ & $(0.3,0.43,0.7)$ & $(0.24,0.34,0.53)$ \\
& 2 & $(0.26,0.34,0.52)$ & $(0.28,0.39,0.62)$ & $(0.25,0.4,0.67)$ & $(0.23,0.37,0.63)$ & $(0.17,0.25,0.47)$ \\
& & & & & & \\
\hline
\end{tabular}

\subsection{Ranking of options}

\begin{tabular}{|c|cc|c|cc|}
\hline & \multicolumn{2}{|c|}{ risk taking } & Neutral & \multicolumn{2}{c|}{ risk averse } \\
\hline $\boldsymbol{\alpha}$ & 0.6 & 0.1 & 1 & 2 & 1.5 \\
\hline $\mathbf{S O}_{\mathbf{1}}$ & 1 & 1 & 3 & 4 & 4 \\
\hline $\mathbf{W O}_{\mathbf{1}}$ & 4 & 2 & 1 & 1 & 1 \\
\hline $\mathbf{S T}_{\mathbf{1}}$ & 3 & 3 & 4 & 2 & 2 \\
\hline $\mathbf{S T}_{\mathbf{2}}$ & 2 & 5 & 2 & 3 & 3 \\
\hline $\mathbf{W T}_{\mathbf{1}}$ & 5 & 4 & 5 & 5 & 5 \\
\hline
\end{tabular}


It's clear that in state of decision making with risk, strategy $\mathrm{SO}_{1}$ and in state of decision making without risk, strategy $\mathrm{WO}_{1}$ have been selected as the best strategies.

\section{Conclusion}

In this paper, we presented a multi-criteria fuzzy group decision - making method for development of proper strategies in an organization and used linguistic variables to estimate of the relative ranking of each option than each criterion. Because we determined uncertainty in criteria and the DM's comments, so we reach to better and more accurate results than the similar. Another advantage this paper is that we could determin mental characteristics of decision makers Using OWA method, while other MCDM methods don't have this ability.

\section{References}

[1] M. Kurttilla , M. Pesone, "Utilizing the analytic hierarchy process (AHP) in SWOT analysis-a hybrid method and its application to a forest-certification case", Forest policy and Economics, 12 (2000) 141-152.

[2] L. Swanson, "Linking maintenance strategies to performance", Intenatonal Journal of production Economics, 8 (2001) 237-244.

[3] R.G. Dyson, "Strategic development and SWOT analysis at the university of Warwick", European Journal of operational research. 10 (2004) 631-640.

[4] I. Yuksel, M. Dagdeviren, "Using the analytic network process (ANP) in a SWOT analysis", Information sciences 19 (2007) 3364-3382.

[5] S. Mehmet, A. Oztekin, "Development of a fuzzy ANP based SWOT analysis for the airline industry in Turkey", Expert Systems with Applications, Volume 39, Issue 1, 11 (2012). 14-24. 